\title{
Filtragem Direcional Radial e Balanceamento Espectral para Atenuação do Groundroll
}

\author{
Beatriz O. de Almeida, CPGG/IGEO/UFBA, Leonardo M. Batista, Wilker Eduardo Souza e Milton J. Porsani \\ CPGG/IGEO/UFBA \& INCT-GP/CNPq/Brasil
}

Copyright 2016, SBGf - Sociedade Brasileira de Geofísica.

Este texto foi preparado para a apresentação no VII Simpósio Brasileiro de Geofísica, Ouro Preto, 25 a 27 de outubro de 2016. Seu conteúdo foi revisado pelo Comitê Técnico do VII SimBGf, mas não necessariamente representa a opinião da SBGf ou de seus associados. É proibida a reprodução total ou parcial deste material para propósitos comerciais sem prévia autorização da SBGf.

\section{Abstract}

The attenuation of the groundroll is one of the main challenges in the land seismic processing. In order to attenuate such noise, the filtering of the seismic data is imperative, since these methods mitigate such problems and therefore increase the signal to noise ratio. In this paper, we apply the Spectral Balance method and the directional radial filter to the attenuation of the groundroll. Numerical results obtained with a seismic line of the Parnaíba Basin illustrate the performance of the proposed methodology.

\section{Introdução}

Foi feito o processamento de parte da linha sismica regional, RL001-09 da Bacia do Parnaíba, adquirida com fonte vibroseis pela empresa Global Geophysical Services Incorporated e disponibilizados pela BP (British Petroleum) no ano de 2015 para pesquisas acadêmicas como parte do projeto PABID.

Com o objetivo de melhorar a razão sinal/ruído, a filtragem de dados sísmicos é uma das principais etapas do processamento já que, a mesma, tem como objetivo atenuar os ruídos, a fim de retratar a imagem de subsuperfície mais próxima possível da geologia real da bacia. Para tanto, é necessário que o geofísico responsável pelo processamento se preocupe em estudar as características do ruído que se deseja atenuar. Caso não ocorra tratamento adequado, refletores serão camuflados na seção sísmica empilhada. Com isso, se faz necessária uma análise cuidadosa para que se evitem eliminações abruptas em partes significativas de bandas do sinal como as reflexões e a criação de artefatos numéricos. Caso consigamos realizar tal etapa de forma adequada, o resultado será um aumento considerável da razão sinal/ruído, proporcionando uma seção empilhada de boa qualidade. Neste trabalho, utilizaremos as técnicas de balanceamento espectral combinado com a filtragem direcional radial.

O balanceamento é um procedimento muito utilizado na atenuação de ruídos com determinada frequência. Desta forma, este método será eficiente para atenuar o ruído coerente denominado groundroll (Ylmaz 1987). Este é dito coerente pelo fato de se conhecerem suas características de baixas frequências e alta amplitude, além de estar relacionado com a propagação de ondas superficiais Rayleigh. Logo, a aplicação da técnica de Balanceamento Espectral resulta em um nivelamento do espectro de frequência, podendo ser aplicadas em dados pré-empilhados ou pós-empilhados, melhorando a resolução temporal e espacial do dado. A decomposição espectral de dados sísmicos ajuda, por exemplo, na análise de estruturas estratigráficas ou reservatórios fraturados.

Já a filtragem direcional radial atenua os eventos lineares, para tanto se faz necessário a escolha de um foco, que no presente caso é escolhido como sendo a posição da fonte. O método utiliza as derivadas ao longo de direções radiais tomadas com relação a um foco, desta forma, todos os ruídos com um determinado padrão radial e linear, como o groundroll, serão atenuados. A combinação desse dois métodos proporciona realces consideráveis nas reflexões que antes estavam mascaradas pelo groundroll. Propusemos os seguintes objetivos: a primeira delas é a atenuação do groundroll existente no dado. Nosso segundo objetivo é amenizar os efeitos da onda aérea na nossa seção. E, por fim, alcançar uma seção sísmica empilhada, que possa ser usada no trabalho de interpretação. Para tais finalidades, utilizamos o software SeisSpace e o Seismic Unix.

\section{Balanceamento Espectral}

Para tratarmos o conceito do Balanceamento Espectral são necessárias definições prévias acerca do Filtro de Frequência. Utilizaremos o filtro passa banda, denominado como trapezoidal. Tal processo define uma faixa de frequência que será preservada, outra que será eliminada e ainda uma região conhecida como slope, na qual a frequência será atenuada. Ou seja, iremos selecionar diversos polígonos de corte no nosso espectro de frequência. $O$ objetivo de tal filtragem é remover os componentes de frequência indesejados e manter o restante do conteúdo sem alterá-los. Desta forma se faz necessária a análise dos intervalos de frequências nos quais estão definidos o groundroll, a onda aérea e os demais ruídos que ocorram durante a aquisição (presentes nas frequências maiores do que $60 \mathrm{~Hz}$, por exemplo). Esse tipo de filtragem é feita no domínio da frequência, ou seja, a transformada de Fourier é necessária antes do processo e sua transformada inversa no fim.

O geofísico que irá processar tal dado deve especificar as frequências que se deseja balancear. Logo, por consequência do processo, se amplifica as altas frequências e atenua as mais baixas frequências. No caso deste trabalho temos como objetivo atenuar o groundroll, desta forma, precisamos balancear as baixas frequências. 
O método que foi utilizado é a forma mais comum do balanceamento espectral. Podemos analisar os passos utilizados através do fluxograma da Figura 1. Inicialmente o dado é convertido do domínio do tempo $\mathrm{x}(\mathrm{t})$, para o domínio da frequência, através da transformada de Fourier 1D. Em seguida o dado é separado em bandas de frequência onde cada um dos traços originais é decomposto em diversos traços com distintos conteúdos de frequência. Tal divisão é realizada como definido acima, pela filtragem passa-banda. Realiza-se a transformada inversa de Fourier a fim de retornar o dado para o domínio do tempo. Após esta etapa, é aplicada uma função de ganho do tipo Automatic Gain Control (AGC) em cada um dos traços decompostos. O usuário pode escolher o número de faixas de banda deste filtro. No presente trabalho, foram escolhidas sete bandas de frequência. Após a equalização, o traço balanceado é obtido pela soma dos traços decompostos em cada banda de frequência.

\section{Filtragem direcional radial (FDR)}

O filtro direcional radial tem a propriedade de atenuar eventos com padrões lineares em determinadas direções, com o objetivo de eliminar as consequências desses na seção sísmica empilhada (Melo et al, 2008). Ou seja, tal filtro será utilizado na detecção de elementos lineares orientados em direções específicas, como o groundroll, a fim de atenuá-los. Para tanto, se faz necessário calcular a derivada na direção de todas as amostras, a partir de operadores como o da Figura 2. Tais operadores de derivadas irão considerar o método de diferenças finitas, que consiste em uma aproximação numérica para as derivadas em meios discretos (Melo et al, 2008). $\mathrm{Na}$ pratica, é necessário que se faça a escolha de um foco no sismograma em domínio de tiro. Após alguns testes observa-se que a posição que produz os melhores resultados são as do ponto de origem do ruído, de forma a obtermos coordenadas $(x 0, t 0)$ na matriz em questão. Nota-se que tais coordenadas encontram-se na parte superior central do sismograma. É observado que onde há coerência nas amplitudes, o efeito é uma atenuação desses eventos e onde há contraste de amplitudes ocorre uma amplificação do sinal, aumentando a razão sinalruido. Desta forma, a FDR corresponderá às derivadas do sismograma ao longo das direções lineares associadas ao ground-roll.

\section{Metodologia e Resultados}

Tendo como objetivo principal a atenuação do groundroll utilizamos os métodos de Balanceamento Espectral e o método de derivada direcional radial. Para obtenção dos resultados finais utilizou-se os softwares SeisSpace e Seismic Unix (SU).

No Seisspace, foi realizada a etapa do pré-processamento dividida em: importação do dado em SEG-Y; geometria; correção estática; filtragem. A etapa de filtragem foi ramificada entre os filtros de frequência, filtro FK, correção de amplitude e balanceamento espectral, afim de realizar comparações iniciais e avaliar qual dos métodos de filtragem resultaria em uma melhor resolução do sinal. Após tais processos inciamos o processamento avançado, no qual dispôs de: análise de velocidade; correção nmo; empilhamento e correção estática residual. Ainda na etapa do pré-processamento observou-se um resultado satisfatório após aplicação do Balanceamento Espectral, já que o mesmo resultou em aumento significativo da razão sinal ruido. Podemos analisar tais resultados a partir da Figura 4 quando comparamos com a Figura 3 do dado bruto. No TV Spectral Whitening, dividimos nosso dado em sete faixas de frequências a fim de atenuar os afeitos causados pelo Groundroll, finalmente, aplicou-se o AGC. O resultado de tais processos foi satisfatório e o método de filtragem a partir do balanceamento espectral se mostrou bastante eficiente, vide Figura 4, tanto na atenuação do groundroll, quanto em revelar as reflexões que se encontravam mascaradas pelo ruído coerente, ou seja, eventos de baixas frequências. Desta forma, obtevese uma maior continuidade das reflexões. O resultado é um realce considerável no sinal na faixa entre 15 e $40 \mathrm{~Hz}$ na seção filtrada com Balanceamento segundo o espectro de frequência. Em contrapartida não conseguimos atenuar a onda aérea.

A segunda etapa foi realizada na plataforma $\mathrm{SU}$, onde transformamos o dado no formado su para acesso direto. Essa transformação é feita para que possamos aplicar o método FDR, desenvolvido em linguagem Fortran. Neste caso o foco foi escolhido na posição do afastamento nulo, central das famílias de ponto de tiro comum no arranjo split-spread, representado na Figura 3 . Foram feitos dois testes, onde realizamos a FDR no dado bruto, seguida do balanceamento espectral. Os resultados estão apresentados nas figuras 5 e 6 , respectivamente. A fim de compará-los, observa-se que a combinação dos dois métodos promoveu um realce significativo nas reflexões que estavam mascaradas pelo ruído groundroll.

A FDR, balanceamento espectral e FDR seguida do balanceamento espectral foi aplicada nos dados préempilhados. Adicionalmente, após o empilhamento, aplicamos a correção estática residual, na qual corrige os erros de deslocamento ocorridos após a correção estática de refração e reflexão, aplicou-se também F-X Decon e filtragem passa-banda com o intuito de realçar ainda mais as reflexões no dado já empilhado.

Quando analisamos a seção sísmica empilhada sem filtragem, o groundroll é observado na forma de eventos lineares com inclinações para ambos os lados, prejudicando o mapeamento dos refletores sísmicos (Figura 3). Já na seção filtrada, com esses dois métodos aplicados, observa-se um aumento significativo na razão sinal/ruído. Podemos observar o resultado da seção empilhada com o Balanceamento espectral na Figura 8 e seção empilhada com o método FDR na Figura 9. O aumento significativo pode ser confirmado quando comparamos tais seções empilhadas com a seção do dado bruto como na Figura 7. Observa-se na figura 10, na qual apresenta a seção empilhada com a combinação dos dois filtros trabalhados, um resultado final satisfatório, a seção empilhada apresenta refletores mais contínuos e bem definidos quando comparado com o dado bruto. 


\section{Conclusões}

Apresentou-se uma aplicação tradicional do método de balanceamento espectral combinado com a técnica de derivada direcional radial. Tal combinação proporcionou uma boa alternativa na filtragem de eventos lineares e para melhoria da razão sinal/ruído. Além disso, houve melhorias significativas nas continuidades e na resolução das reflexões, quando obtivemos 0 dado empilhado. Já que a combinação desses métodos revelou as reflexões que antes se encontravam mascaradas pelo ruído coerente. Logo, conclui-se que, as seções sísmicas filtradas com a combinação de tais métodos geraram refletores susceptíveis à interpretação geológica.

\section{Agradecimentos}

Os autores agradecem à BP/PABIP, INCT-GP/CNPq/MCT, PETROBRAS, pelo financiamento e apoio a pesquisa. Também agradecemos à LANDMARK pela licença do software SeisSpace cedida ao CPGG/UFBA

\section{Referências}

Santos, S. A. (2014). Atenuação do ground-roll utilizando a filtragem directional radial. Trabalho de Conclusão de curso. Universidade Federal da Bahia, Salvador, Brasil.

Dobrin, M. B.; Lawrence, L. P. and Sengbush, R. L. (1954). Surface and near-surface waves in the Delaware Basin, Geophysics.

Grant, F. S. and West, G. F. (1965). Interpretation theory in applied geophysics, Society of Exploration Geophysicists.

Melo, P.E.M., M.J. Porsani and Silva, M.G. (2008). Groundroll attenuation using a 2D time-derivate filter. Geophysical Prospecting, 57, 343-353.

Parasnis, D. S. (1997). Principles of applied geophysics, Chapman \& Hall, $5^{a}$ edic.

Silva, M. G. (2004). Processamento de dados sísmicos da bacia do Tacutu, Dissert. De Mestrado. Universidade Federal da Bahia, Salvador, Brasil.

Yilmaz, O. (1987). Seismic data processing, Society of Exploration Geophysicists, Tulsa - Oklahoma.

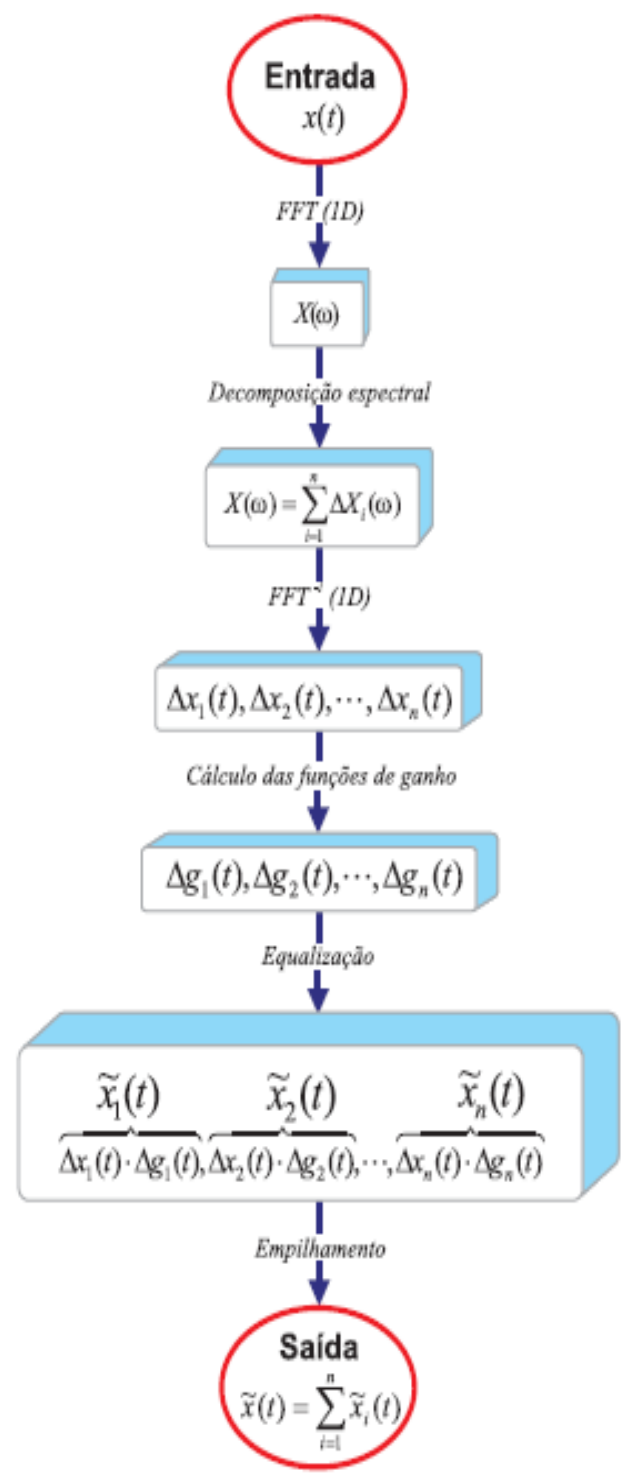

Figura 1: Fluxograma utilizado para aplicação do Balanceamento Espectral (Adaptada de Silva (2009)).

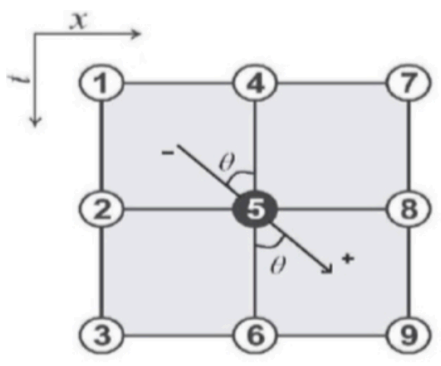

Figura 2: Operador de derivada direcional $(3 \times 3)$ (Adaptada de Santos, (2014)). 


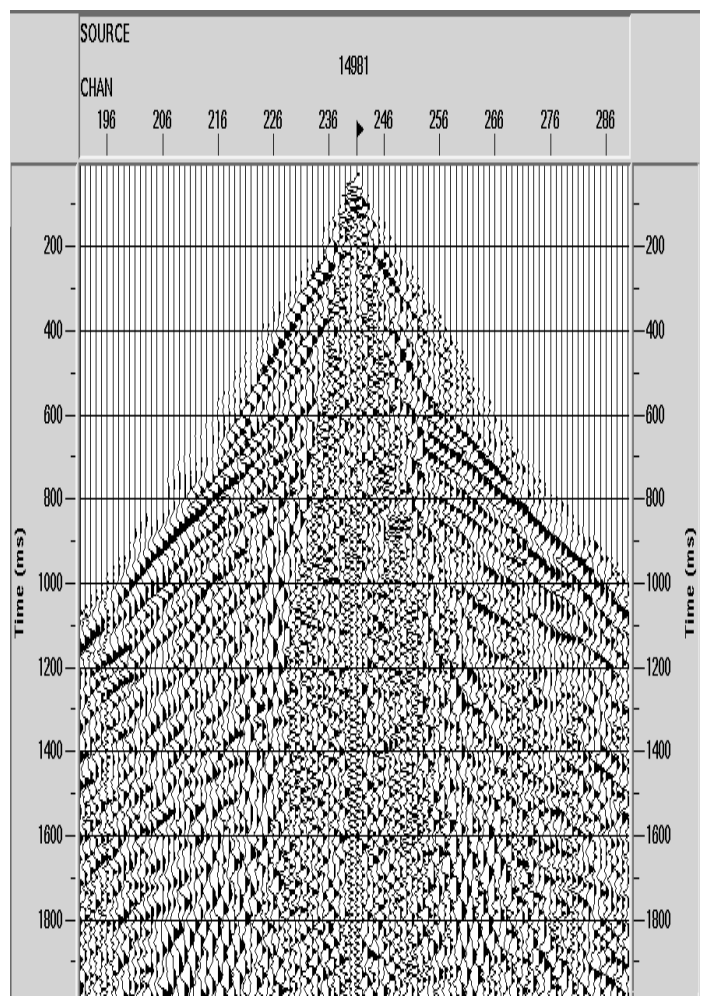

Figura 3: Família de traços de ponto de tiro comum.

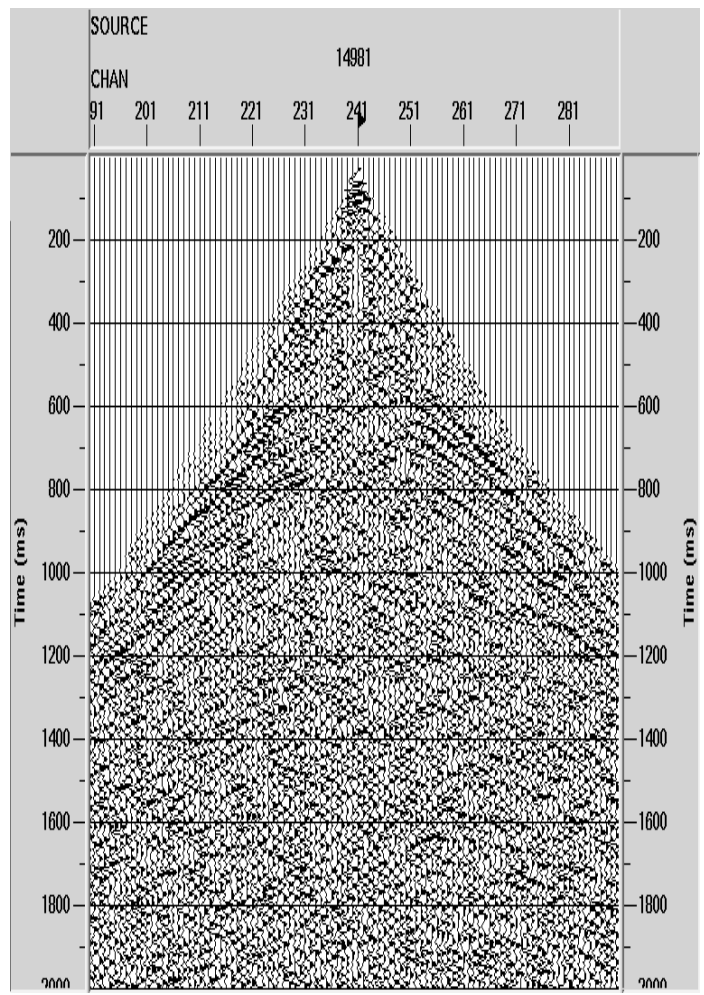

Figura 4: Resultado da aplicação do balanceamento espectral aos dados da Figura 3.

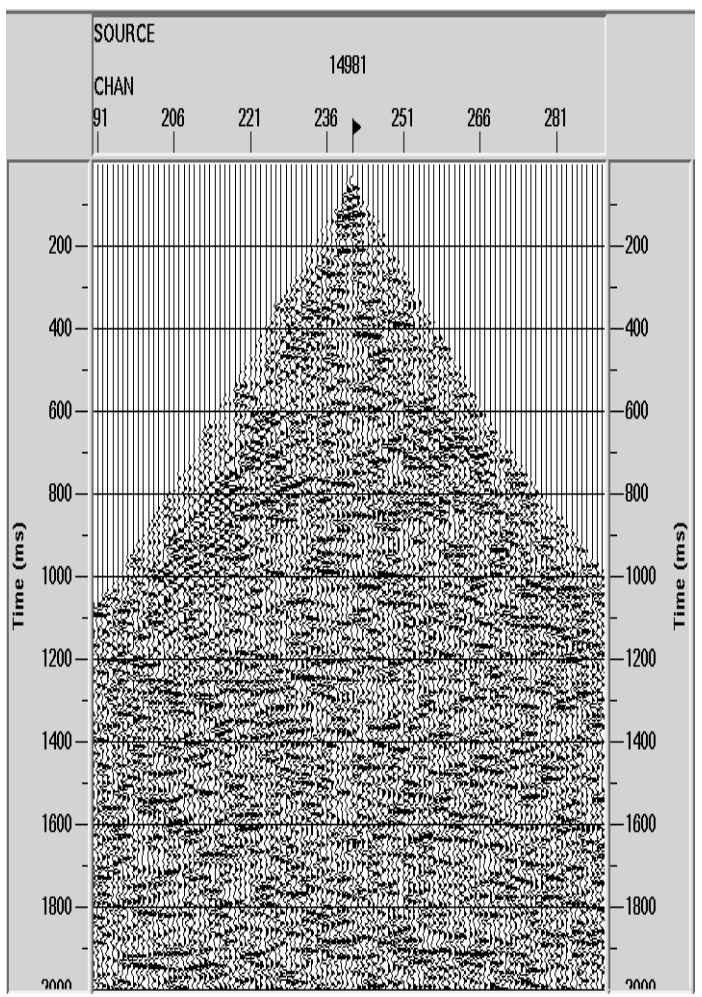

Figura 5: Aplicação da filtragem de derivada direcional radial aos dados da Figura 3.

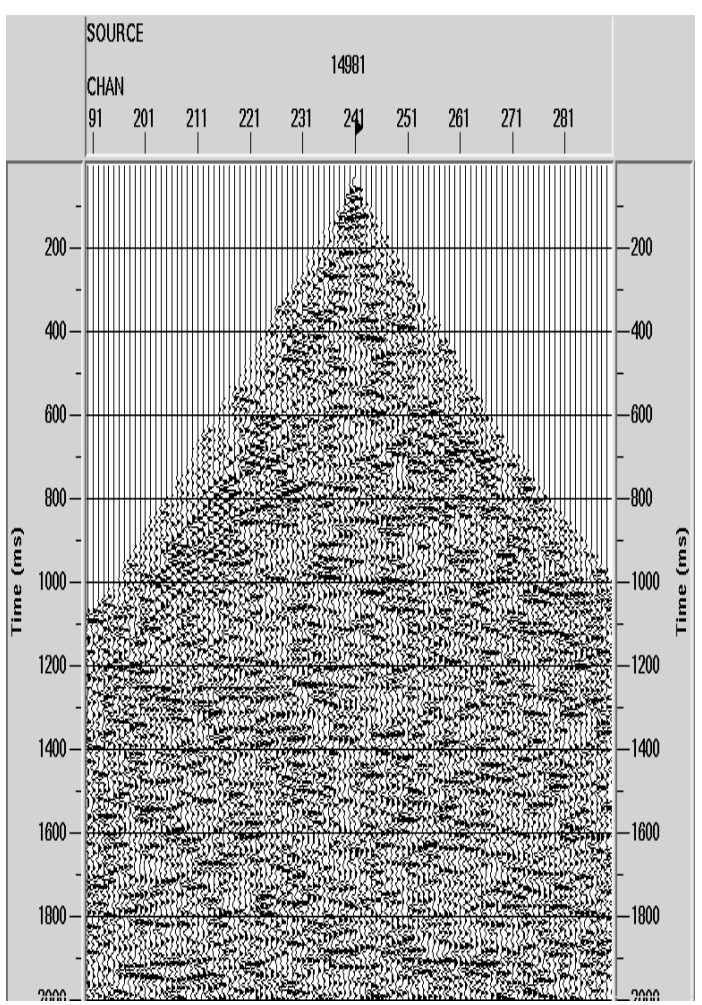

Figura 6: Aplicação do balanceamento espectral aos dados da Figura 5. 


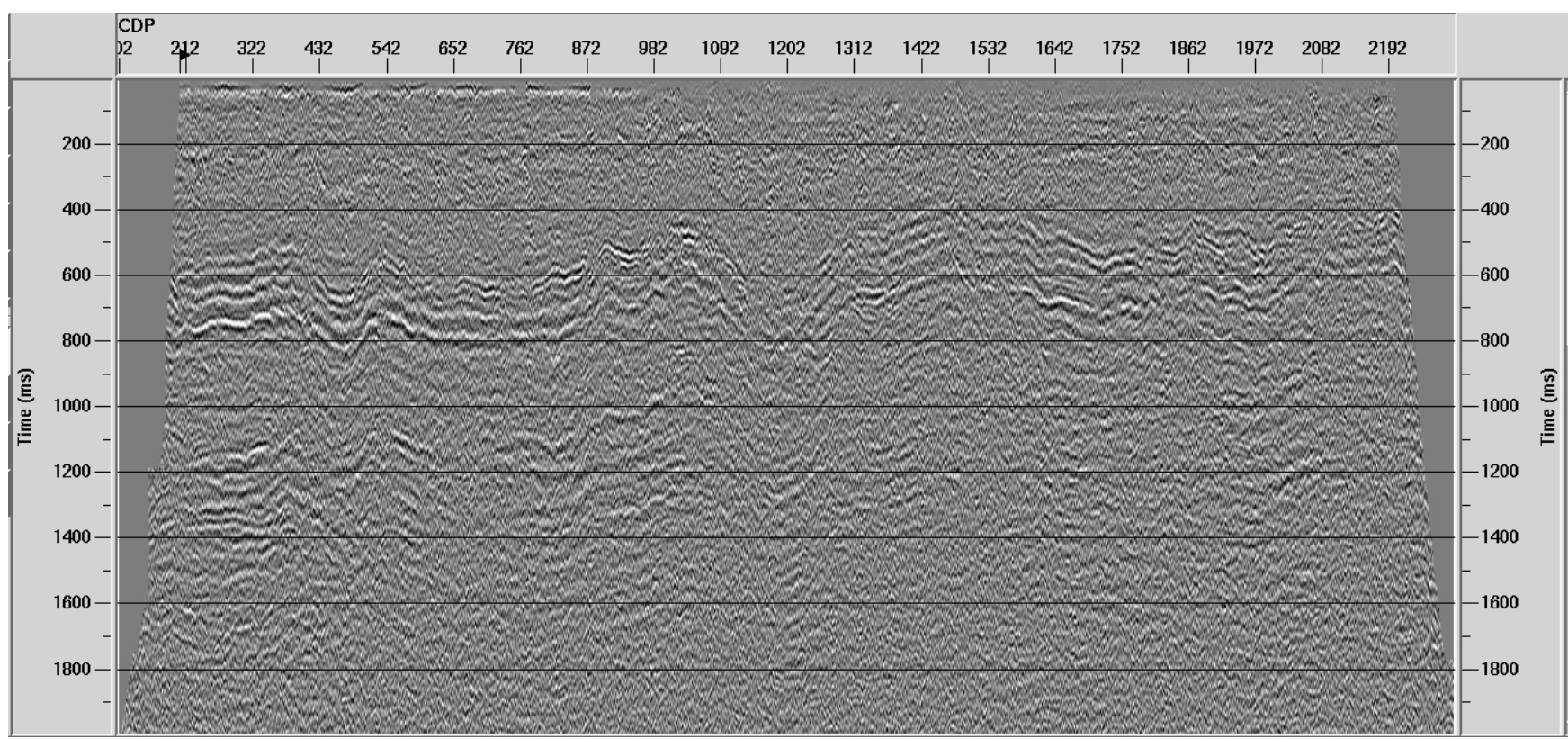

Figura 7: Seção empilhada do dado bruto.

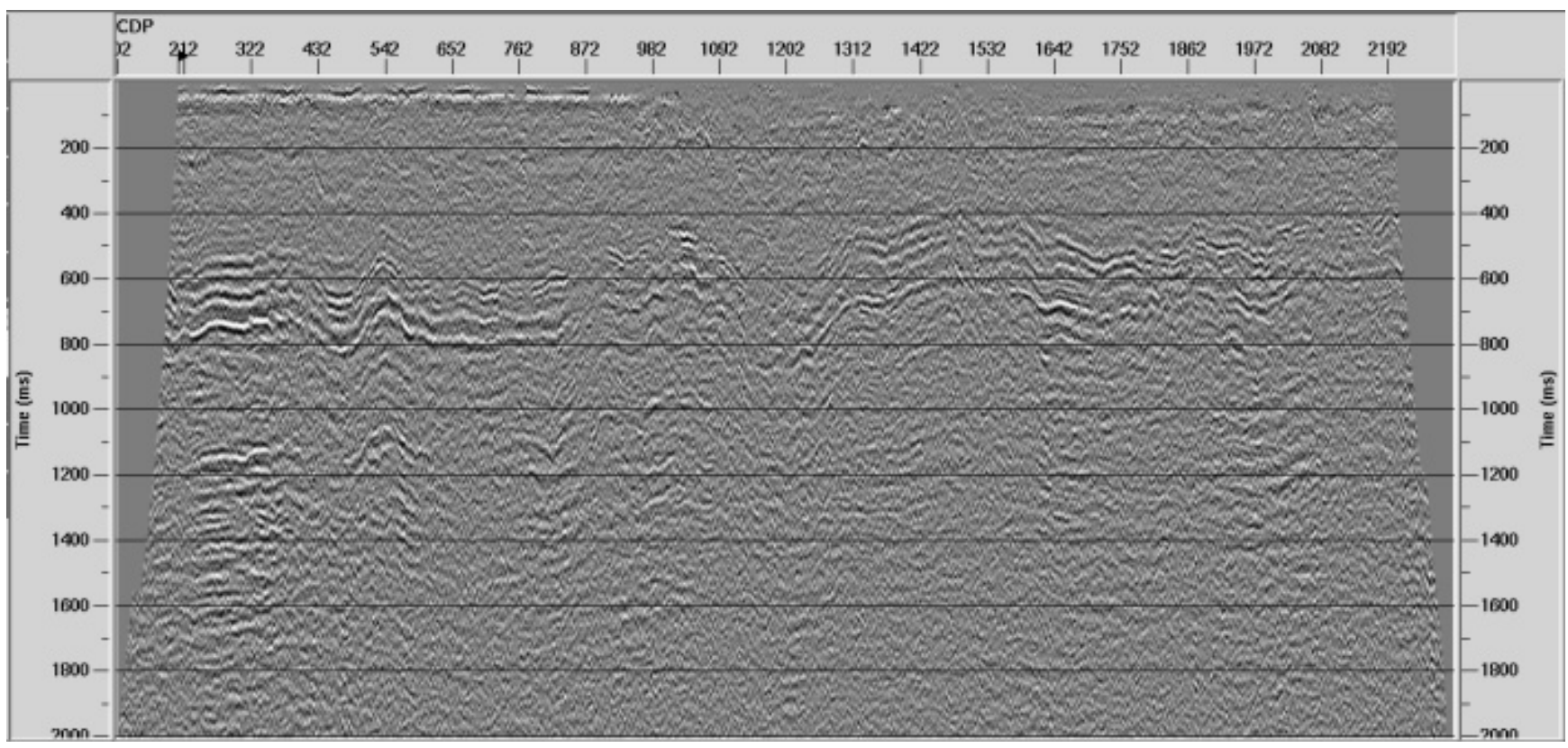

Figura 8: Seção empilhada após aplicação do Balanceamento Espectral. 


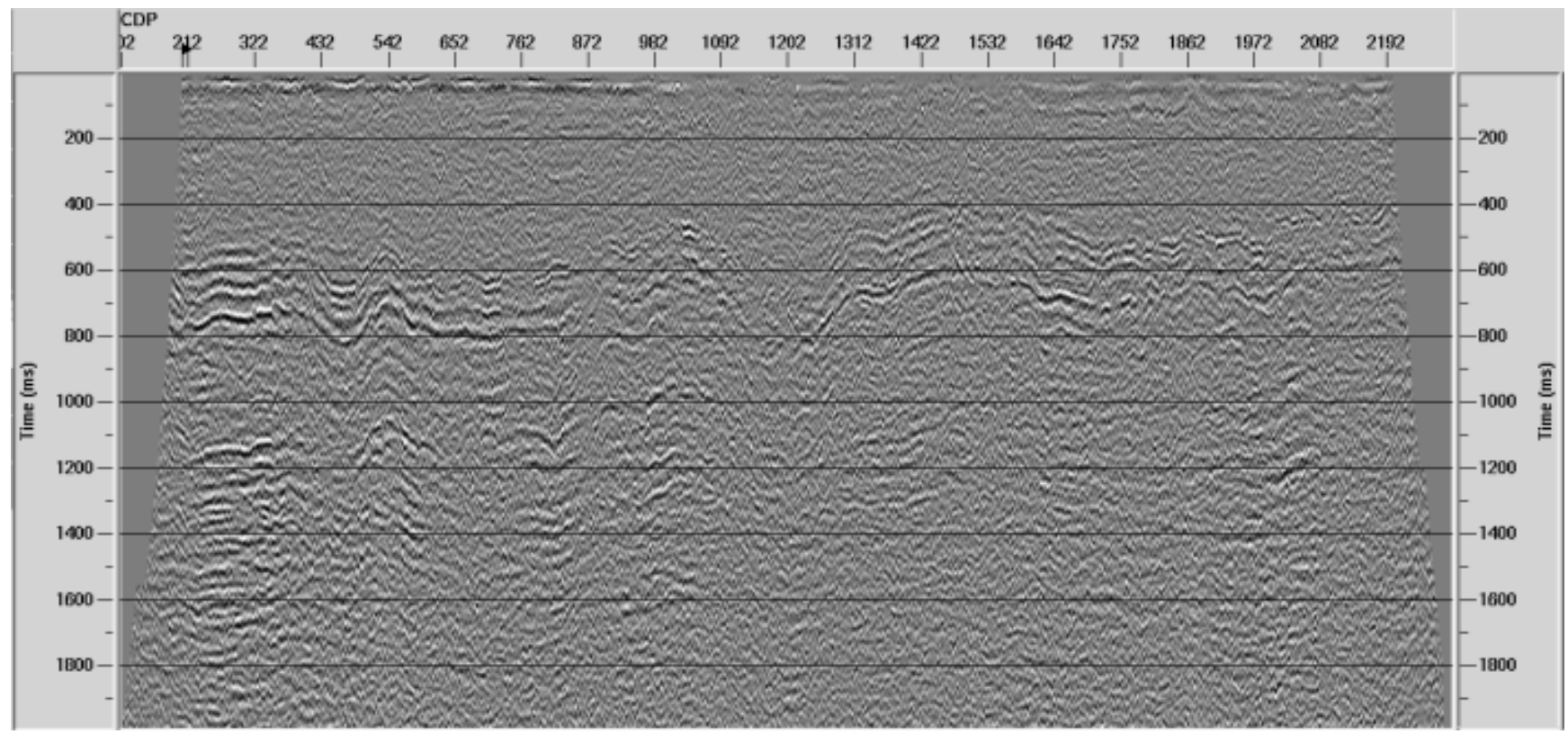

Figura 9: Seção empilhada após aplicação da filtragem direcional radial (FDR).

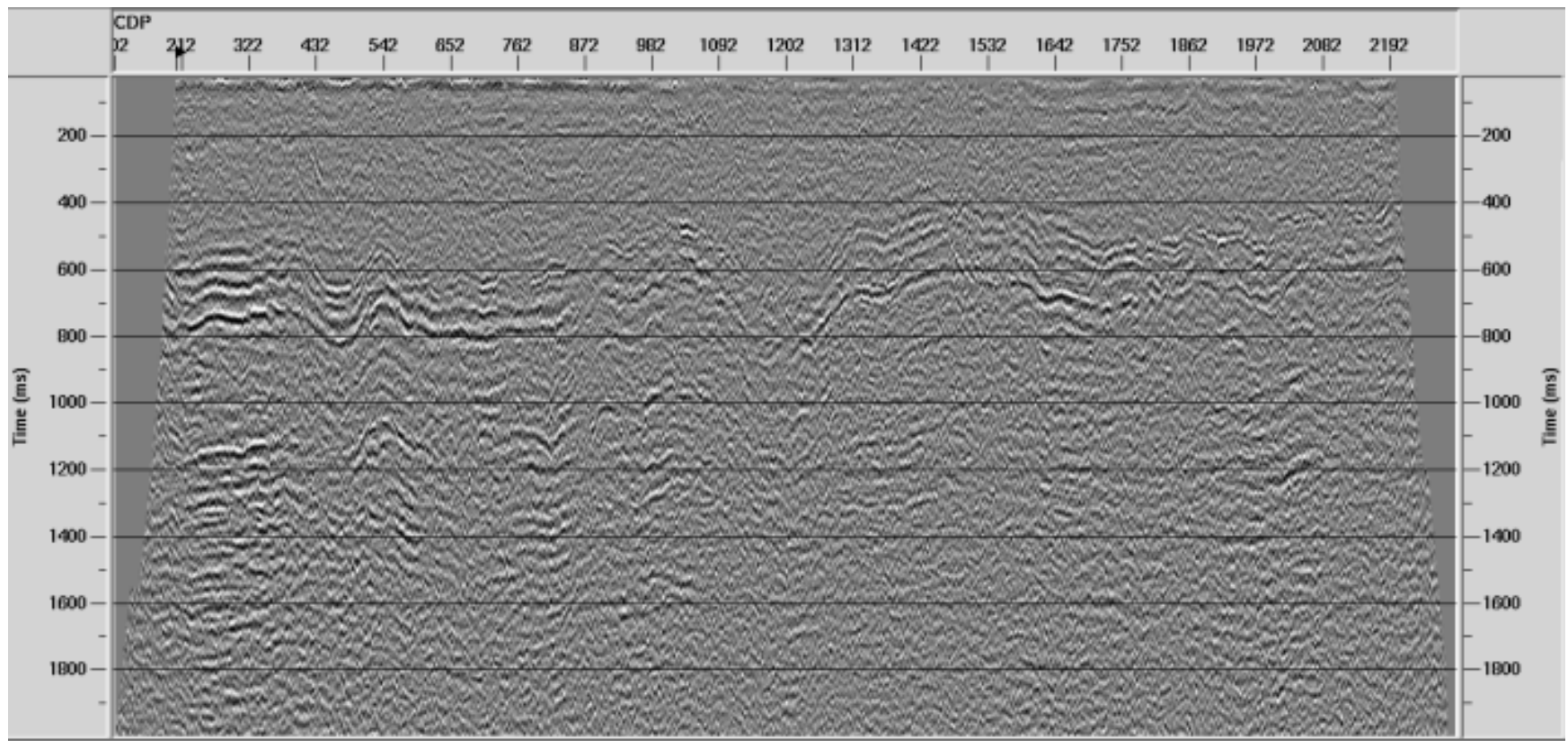

Figura 10: Seção empilhada após aplicação do Balanceamento Espectral seguido da filtragem direcional radial. 